\title{
High-resolution transversal load sensor using a random distributed feedback fiber laser
}

\author{
V. DeMiguel-Soto*, D. Leandro, M. Lopez-Amo \\ Universidad Pública de Navarra, Dept. of Electrical and Electronic Engineering, Campus de \\ Arrosadia S/N, E-31006, Pamplona, Spain \\ *veronica.demiguel@unavarra.es; phone +34 94816 9841; fax +34948 16 9720;
}

\begin{abstract}
In this work, a new application of random distributed feedback lasers to fiber optic sensing has been presented. The particular properties of these lasers, such as the lack of longitudinal modes and high stability, have been exploited to monitor transversal load using a phase-shifted fiber Bragg grating (PS-FBG), obtaining a resolution of $1 \mathrm{~g}$ and a sensitivity of $3.95 \mathrm{GHz} / \mathrm{Kg}$. Due to the PS-FBG birefringence and the load-interrelated transmission lines generated by the PS-FBG along the orthogonal polarization directions, the beating of the two emission lines generated in the laser can be monitored in the electrical domain. As a result, transversal load applied on the sensor can be measured.
\end{abstract}

Keywords: random distributed feedback laser, phase-shifted fiber Bragg grating (PS-FBG), beating, transversal load, high resolution

\section{INTRODUCTION}

Random distributed feedback (DFB) fiber lasers generated by means of the amplified backscattered light in single-mode fiber (SMF) have been lately proposed by Turitsyn et al. [1]. The backscattered light caused by the Rayleigh scattering effect along kilometers of SMF provides this feedback and the stimulated Raman scattering effect generates the amplification process. Several works have analyzed the particular properties of these fiber lasers, such as their lower noise figure, mode-less behavior, absence of mode competition and mode hopping. Although just a few works have been proposed recently [2]-[5], random DFB lasers have a promising future in the optic sensing field.

Fiber Bragg gratings (FBGs) have been studied intensively during the last years due to their wide variety of industry applications, such as components in fiber-optics communication systems and many kinds of fiber-optics sensors. However, classical monitoring techniques based on the analysis of the optical spectrum offer limited resolution. As a consequence, frequency beating techniques using dual-wavelength continuous wave lasers in conjunction with FBGs are now being explored as a possibility to improve the resolution attained. Some interesting approaches to monitor physical properties like liquid-level [6], strain, temperature [4] and simultaneous strain and temperature [7] have been carried out using this beating technique. Moreover, transversal loading sensors have been demonstrated [8]-[9] achieving a maximum sensitivity of $1.42 \mathrm{GHz} / \mathrm{Kg}$ in [9]. Despite that, this system is not able to demonstrate high resolution measurements due to the intermodal distance given by the cavity length, which limits the resolution of such fiber laser systems.

In this work, the beating of two narrow-linewidth emission lines of a random DFB fiber laser is monitored to obtain high-resolution transversal load measurements, with a sensitivity of $3.95 \mathrm{GHz} / \mathrm{Kg}$ and a resolution of $1 \mathrm{~g}$. The modeless behavior of the laser provides high frequency stability and eliminates instabilities related to mode hopping and mode competition, which are present in previous approaches.

\section{EXPERIMENTAL SETUP AND PRINCIPLE OF OPERATION}

\subsection{Experimental setup}

The presented setup (Fig. 1) is based on a forward-pumped random distributed feedback (DFB) fiber laser. A wavelength division multiplexer (WDM) is used to inject the pump laser (IPG RLD 3-1445) into the cavity formed by 50 kilometers

Sixth European Workshop on Optical Fibre Sensors, edited by Elfed Lewis, Proc. of SPIE Vol. 9916, $991606 \cdot$ • (c) 2016 SPIE · CCC code: 0277-786X/16/\$18 · doi: 10.1117/12.2236195 
of TrueWave single mode fiber spool (TW-SMF). This fiber performs two important roles: on one hand it is used as the active medium for the amplification due to the stimulated Raman scattering effect. On the other hand, it acts as the distributed mirror required for the laser generation, providing a weak feedback along the fiber because of the Rayleigh scattering effect. The back scattered light in the fiber enters into a loop mirror by the port 2 of a 3-port optical circulator, is filtered by a programmable filter and a phase-shifted Bragg grating (PS-FBG), which acts as a filter and a sensor at the same time. Finally the light is redirected again into the cavity. An optical isolator is placed at the fiber end to avoid any reflection or undesired feedback from the output port to the laser's cavity. Another WDM is disposed after the isolator to extract the pump wave remaining at the end of the fiber. A polarization controller and a polarizer have been used in order to adjust the power of the two emission lines and enhance the beating between them. The signal has been firstly monitored in the optical domain using a BOSA (Brillouin Optical Spectrum Analyzer) and also has been filtered, detected by a $12 \mathrm{GHz}$-bandwidth photodetector and finally monitored on an Electrical Spectrum Analyzer (ESA). A variable attenuator was used at the output of the laser to limit the power received and protect the devices.

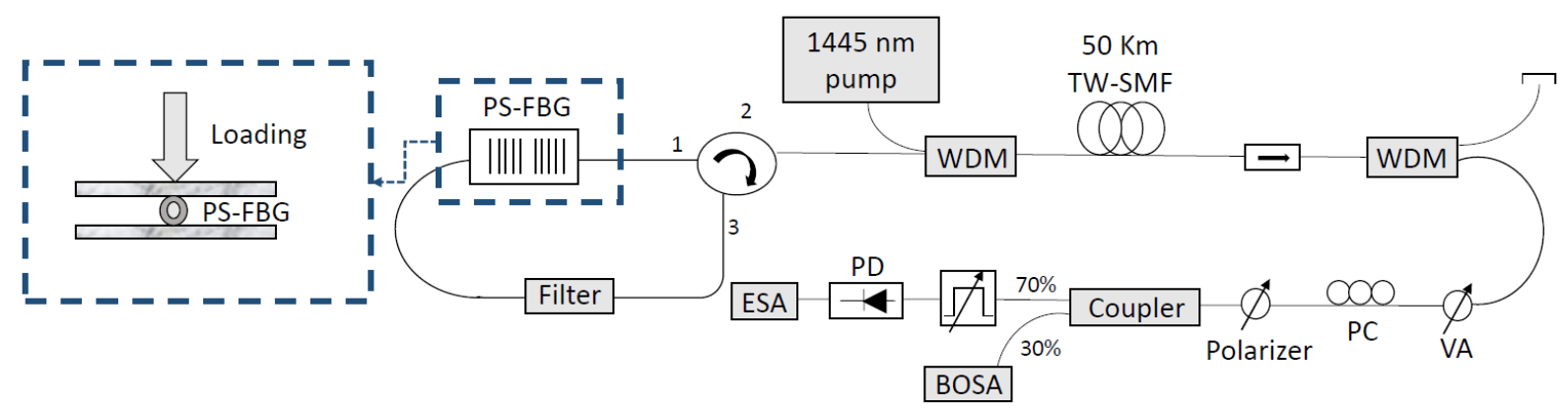

Figure1. Schematic diagram of the proposed laser for transversal load measurements; inset is the loading configuration.

\subsection{Sensing principle}

Typically, PS-FBGs present two transmission peaks due to the different refractive indexes along the orthogonal directions. When a transverse force is applied to the sensor, those transmission lines will separate due to the change in the PS-FBG birefringence. The frequency spacing between the two transmission peaks along the two orthogonal polarization states is given by [10]:

$$
\Delta v=v_{x}-v_{y}=\frac{\mathrm{c} \cdot \mathrm{B}}{\mathrm{n}_{0} \cdot \lambda_{0}}
$$

Where $n_{0}$ is the average index of the fiber, $\lambda_{0}$ is the Bragg wavelength of the fiber grating and $B$ is the load-induced birefringence, given by

$$
B=\frac{2 \cdot n_{0}{ }^{3} \cdot\left(p_{11}-p_{12}\right)\left(1+v_{p}\right) \cos (2 \theta) \cdot F}{\pi E r}
$$

And $p_{11}$ and $p_{12}$ are the components of the strain-optical tensor of the optical material, $v_{p}$ is the Poisson's ratio, $E$ is the Young's modulus of the fiber, $\theta$ is the angle between the direction of the force and the polarization axis of the fiber, $r$ is the radius of the fiber and $F$ is the linear transverse load (force per unit length). Thanks to the load-interrelated transmission lines along the orthogonal polarization directions, two emission lines are generated at different frequencies. The beating between these two signals will generate a third signal whose frequency is a function of the load induced birefringence. As a result, the transverse load applied to the PS-FBG can be determined by measuring the beat frequency.

\section{EXPERIMENTAL RESULTS}

In the experiment, a PS-FBG with transmission peaks located around $1545.3 \mathrm{~nm}$ was used. A programmable filter with a tunable band-pass of $0.1 \mathrm{~nm}$ was used to filter the transmission peaks of the PS-FBG. Initially, the verification of achieving a random DFB fiber laser was carried out. Firstly, Fig. 2(a) shows the abrupt increase of the peak power corresponding to the lasing threshold of the system $(1.32 \mathrm{~W})$. Additionally, the lack of longitudinal modes was verified 
by observing the self-heterodyne signal of the laser in the electrical domain. The optical spectrum of the laser can be seen in Fig. 2(b) measured by means of a BOSA with a pump power of $1.46 \mathrm{~W}$. The peak power obtained is $-13.7 \mathrm{dBm}$ with an optical signal to noise ratio of around $10 \mathrm{~dB}$.
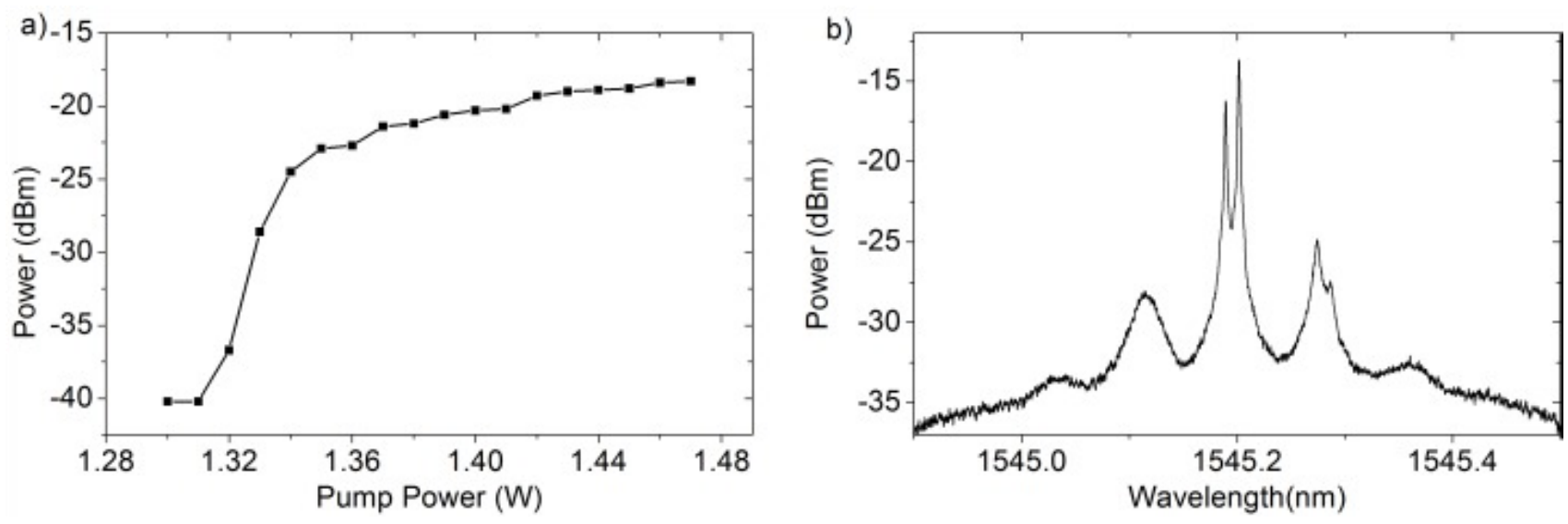

Figure 2. a) Study of the output power vs pump power injected in the system. b) Optical spectrum of the random DFB fiber laser used for transversal load sensing.

After demonstrating the adequate behavior of the system as a random DFB laser, the transversal load measurements have been carried out. When transversal load is applied, the wavelength spacing between the two polarization peaks increases (Fig. 3(a)); consequently, the electrical beating moves to higher frequencies, see Fig. 3(b).
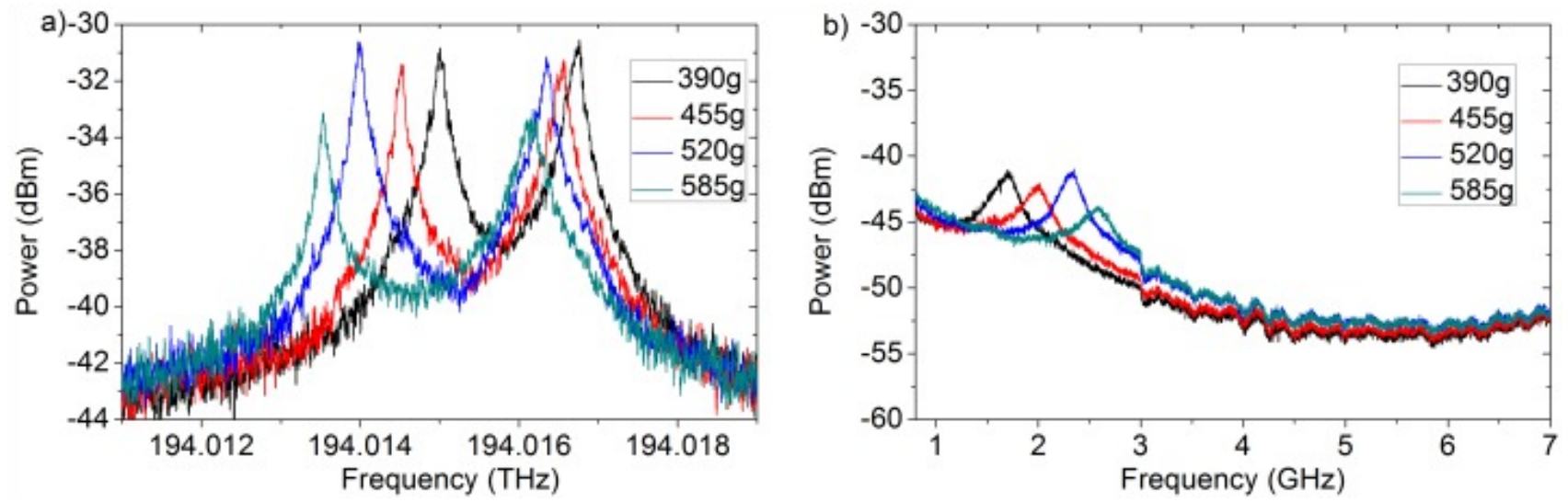

Figure 3. a) Measured optical spectra of the two emission lines and b) measured electrical spectra of the beating signal when different transversal loading is applied.

Thus, it is possible to measure transversal load in both optical and electrical domain. However, measuring in the electrical domain with an ESA has two important advantages. The first is its higher resolution. The second is the simplicity of the measurement process, since the frequency of a single peak has to be monitored. As a consequence, transversal load has been measured following the electrical beating of the emission lines in the ESA. These measurements have been taken at room temperature with a load step of 65 grams. The results, displayed in Fig. 4(a), show a clear linear behavior with a sensor sensitivity of $3.95 \mathrm{GHz} / \mathrm{Kg}$. It can be observed a good linear fitting of the measurements with an error $\mathrm{R}^{2}=0.996$. The main reason of the mismatch with the linear fitting is probably caused by the experimental setup itself. Since the system that applies the load on the PS-FBG was not perfectly settled, possible variations of the angle in which the weight is applied could have caused a small mismatch in the measurements. Accordingly, to quantify the maximum possible resolution of the system, the instability of the laser was measured at room temperature and at a constant load. The beating of the two emission lines was measured every $30 \mathrm{sec}$ for a total period of $30 \mathrm{~min}$. The laser instability results are shown in Fig. 4(b), from which a resolution of $4 \mathrm{MHz}$ can be derived. 

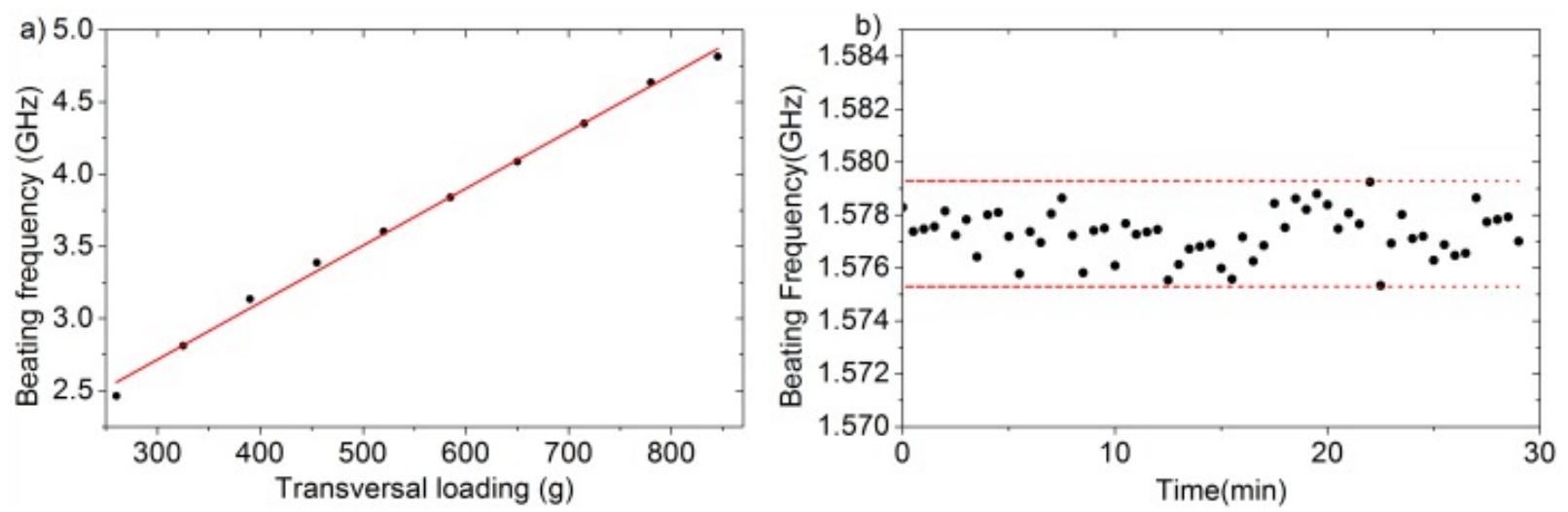

Figure 4. a) Variation of the beating frequency betweenthe two emission lines versus transversal loading. b) Stability measurements of the beating frequency at constant loading during $30 \mathrm{~min}$

\section{CONCLUSION}

In this study, a new sensing application of random DFB lasers has been presented. The particular properties of random DFB fiber lasers have been exploited to interrogate a PS-FBG as a transversal load sensor. In particular, the absence of longitudinal modes of random DFB fiber lasers allows attaining higher resolution than in other transversal load sensing setups (especially over the systems based in erbium doped fiber lasers). In the proposed study, the random laser line is narrowed by means of a programmable filter and a phase-shifted fiber Bragg grating, which filters two narrow loadinterrelated emission lines with orthogonal polarization whose beating reflects the transversal load on the sensor.

\section{ACKNOWLEDGEMENTS}

Financial support from the Spanish Comisión Interministerial de Ciencia y Tecnología within project TEC2013-47264$\mathrm{C} 2-2-\mathrm{R}$ and the FEDER funds are acknowledged.

\section{REFERENCES}

[1] Turitsyn, S., Babin, S., El-Taher, A.E., Harperm P., Churkin, D.V., Kablukov, S.I., Ania-Castañón, J.D., Karalekas, V. and Podivilov, E.V., "Random distributed feedback fibre laser" Nat. Photon. 4(4), 231-235 (2010).

[2] Pinto, A. M. R., Lopez-Amo, M., Kobelke, J. and Schuster, K., "Temperature fiber laser sensor based on a hybrid cavity and a random mirror" J. Lightwave Technol., 30(8), 1168-1172 (2012).

[3] Bravo, M., DeMiguel-Soto, V., Ortigosa, A. and Lopez-Amo, M., "Fully Switchable Multi-Wavelength Fiber Lasers Based on Random Distributed Feedback for Sensors Interrogation” J. Lightwave Technol., 33(12), 2598 - 2604 (2015).

[4] Leandro, D., Ardanaz, D. and Lopez-Amo. M., "High resolution fiber Bragg grating interrogation using a random distributed feedback fiber laser" Proc. SPIE 9634, 24th International Conference on Optical Fiber Sensors, 963400 (2015).

[5] Leandro, D., Perez-Herrera, R., Bravo, M., Lopez-Amo, M., "Time and wavelength division multiplexing scheme for ultra-long sensing based on a cavity-modulated random DFB fiber laser" Proc. SPIE 9634, 24th International Conference on Optical Fibre Sensors, 963415 (2015)

[6] Dai, Y., Sun, Q., Tan, S., Wo, J., Zhang, J., and Liu, D., "Highly sensitive liquid-level sensor based on dualwavelength double-ring fiber laser assisted by beat frequency interrogation" Opt. Express 20, 27367-27376 (2012).

[7] Gao, L., Chen, L., Huang, L., and Chen, X., "Multimode fiber laser for simultaneous measurement of strain and temperature based on beat frequency demodulation" Opt. Express 20, 22517-22522 (2012).

[8] Yu, K., Wu, C., Sun, M., Lu, C., Tam, H. Y., Zhao, Y. and Shao, L., "Fiber laser sensor for simultaneously axial strain and transverse load detection" Measurement, 62, 137-141 (2015).

[9] Fu, H., Shu, X., Mou, C., Zhang, L., He, S., and Bennion, I., "Transversal loading sensor based on tunable beat frequency of a dual-wavelength fiber laser" Photonics Technology Letters IEEE, 21(14), 987-989 (2009).

[10] Guan, B., Jin, L., Zhang, Y. and Tam, H., "Polarimetric Heterodyning Fiber Grating Laser Sensors" J. Lightwave Technol., 30(8), 1097-1112 (2012) 